\title{
CORRECTION
}

\section{Author Correction: CDER167, a dual inhibitor of URAT1 and GLUT9, is a novel and potent uricosuric candidate for the treatment of hyperuricemia}

Ze-an Zhao ${ }^{1}$, Yu Jiang ${ }^{1}$, Yan-yu Chen ${ }^{1}$, Ting Wu ${ }^{1}$, Qun-sheng Lan ${ }^{1}$, Yong-mei Li ${ }^{1}$, Lu Li ${ }^{1}$, Yang Yang ${ }^{1}$, Cui-ting Lin ${ }^{1}$, Ying Cao ${ }^{1}$, Ping-zheng Zhou', Jia-yin Guo ${ }^{1}$, Yuan-xin Tian ${ }^{1}$ and Jian-xin Pang ${ }^{1}$

Acta Pharmacologica Sinica (2022) 43:1884; https://doi.org/10.1038/s41401-022-00872-z

Correction to: Acta Pharmacologica Sinica https://doi.org/10.1038/ s41401-021-00640-5, Published online 25 March 2021

The authors regret that the acknowledgments section of the original manuscript was incorrect. The corrected acknowledgements are shown below.
This work was supported by the National Natural Science Foundation of China (Grant Nos. 81974507 and 81773794) and the Science and Technology Program of Guangzhou City, China (Grant No. 201903010087).

${ }^{1}$ Guangdong Provincial Key Laboratory of Drug Screening, School of Pharmaceutical Sciences, Southern Medical University, Guangzhou 510515, China Correspondence: Jia-yin Guo (g1227@smu.edu.cn) or Yuan-xin Tian (tyx523@163.com) or Jian-xin Pang (pjx@smu.edu.cn)

These authors contributed equally: Ze-an Zhao, Yu Jiang, Yan-yu Chen

Published online: 27 January 2022 